\title{
Wave spectra of square-lattice domains in a quasi-two-dimensional binary complex plasma
}

\author{
H. Huang, ${ }^{1}$ A. V. Ivlev, ${ }^{2}$ V. Nosenko, ${ }^{3}$ Y.-F. Lin, ${ }^{1}$ and C.-R. Du ${ }^{1,4, a)}$ \\ 1) College of Science, Donghua University, 201620 Shanghai, PR China \\ 2) Max Planck Institute for Extraterrestrial Physics, 85748 Garching, Germany \\ ${ }^{3)}$ Institut für Materialphysik im Weltraum, Deutsches Zentrum für Luft- und Raumfahrt (DLR), 82234 Weßling, \\ Germany \\ 4) Member of Magnetic Confinement Fusion Research Centre, Ministry of Education, 201620 Shanghai, \\ PR China
}

(Dated: 2 January 2019)

Domains of square lattice have been observed in a quasi-two-dimensional binary complex plasma. The longitudinal and transverse mode of the wave spectra were measured. To compare with the experiment, Langevin dynamics simulations of a binary mixture were carried out, where the non-reciprocal interactions between different species were modeled with a point-wake Yukawa potential. A strong dependence of the wave spectra on the relative magnitude of the point-wake charge is revealed.

PACS numbers: 52.27.Lw

Keywords: Complex plasma

A complex plasma consists of weakly ionized gas and microparticles. The particles are charged negatively while interacting with ions and electrons. Since the discovery of plasma crystals ${ }^{1-5}$, complex plasmas have been widely used as a model system ${ }^{6-8}$ to study condensed matter physics. In the laboratory, microparticles are levitated in the (pre)sheath above the bottom electrode $^{9-11}$, where the gravity can be balanced by the electric force. Under certain conditions, monodisperse microparticles are confined in a single layer and selforganize in a triangular (hexagonal) lattice ${ }^{12}$. Various phenomena, such as crystallization ${ }^{13,14}$, generation of the Mach cones ${ }^{15-17}$, wave propagation ${ }^{18-20}$, and the mode coupling instability ${ }^{21-23}$, have been observed in $2 \mathrm{D}$ complex plasmas.

A binary complex plasma is formed by injecting two types of particles in a plasma ${ }^{24-26}$. With a proper combination of the particle sizes and materials, the two species can be levitated at approximately the same height, i.e., the distance between the two layers can be much smaller than the horizontal interparticle distance ${ }^{27-29}$. This is called quasi-two-dimensional (q2D) binary complex plasma. The collective dynamics and structure properties of such systems have been studied both in experiments and theory ${ }^{30-32}$.

In this paper, we report an experimental observation of domains of square lattice in a q2D binary complex plasma. Wave spectra of such domain are measured and the results are compared with a numerical simulation.

The experiments were performed in a modified Gaseous Electronics Conference (GEC) rf reference cell $^{17}$, see Fig. 1. Argon plasma was sustained using a capacitively coupled rf discharge at $13.56 \mathrm{MHz}$ with an input power of $20 \mathrm{~W}$. The gas pressure was about

\footnotetext{
a) Electronic mail: chengran.du@dhu.edu.cn
}

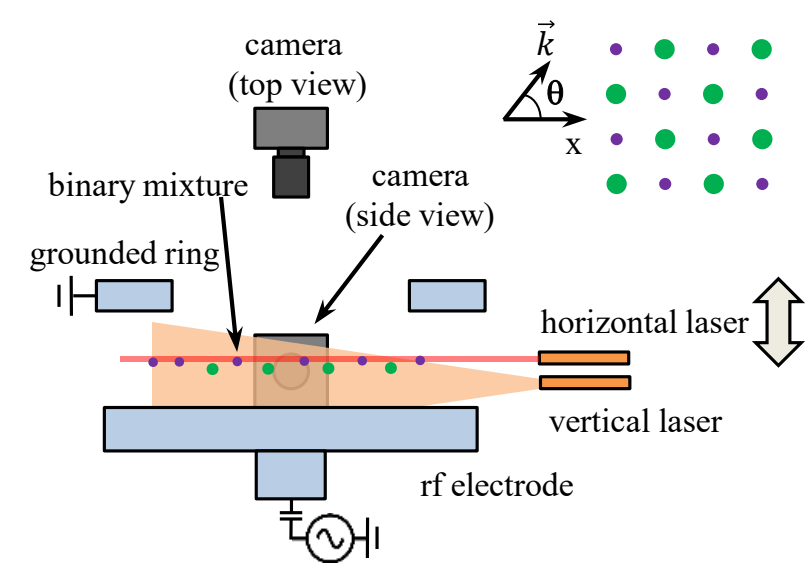

FIG. 1. Sketch of experimental setup and the frame of reference used for the analysis. The plasma is sustained by a capacitively coupled argon discharge in the GEC rf reference cell. For particle diagnostics, the horizontal laser diode can be moved (scanned) in the vertical direction. A binary mixture of particles forms a square lattice with the structure $S(A B)$, the purple circles represent small MF particles and the green circles represent big PS particles.

0.65 Pa. To form a q2D binary complex plasma, we injected monodisperse melamine formaldehyde (MF) and polystyrene (PS) microparticles consecutively into the plasma $^{29}$. These particles were suspended above the bottom circular electrode with a diameter of $220 \mathrm{~mm}$ and confined horizontally in its center by a grounded ring. The MF particles had a diameter of $d_{\mathrm{MF}}=9.19 \pm 0.09 \mu \mathrm{m}$ and mass density of $\rho_{\mathrm{MF}}=1.51 \mathrm{~g} / \mathrm{cm}^{3}$, while the PS particles had a diameter of $d_{\mathrm{PS}}=11.36 \pm 0.12 \mu \mathrm{m}$ and mass density of $\rho_{\mathrm{PS}}=1.05 \mathrm{~g} / \mathrm{cm}^{3}$. The gravity was compensated by the sheath electric force as $m g=E Q$, where $g$ is the acceleration due to gravity, $E$ is the electric field, 

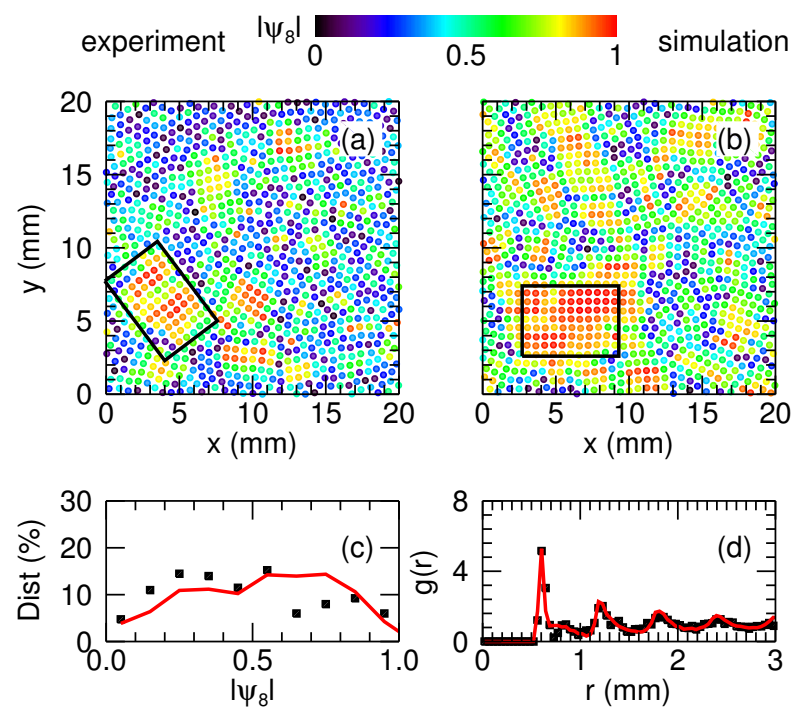

FIG. 2. Local structure indicated by the order parameter $\left|\Psi_{8}\right|$ of a q2D binary complex plasma for experiment (a) and simulation (b), the distribution of $\left|\Psi_{8}\right|$ (c), and the pair correlation function (d). The rectangles in (a) and (b) indicate the areas used for the analysis of the spectra shown in Fig. 4. In (c) and (d), the experimental results are shown by the black squares, while the red lines represent the simulations.

and $Q$ is the particle charge. Assuming $Q \propto d^{33}$, the two types of particles can be suspended at the same height when the condition $\rho_{\mathrm{MF}} d_{\mathrm{MF}}^{2}=\rho_{\mathrm{PS}} d_{\mathrm{PS}}^{2}$ is fulfilled. The corresponding neutral gas damping rates are $\nu_{\mathrm{PS}}=0.91 \mathrm{~s}^{-1}$ and $\nu_{\mathrm{MF}}=0.77 \mathrm{~s}^{-1}$ for PS and MF particles, respectively, following the Epstein expression ${ }^{34-37}$. The particles were illuminated by two laser sheets. The wavelength of the horizontal laser sheet was $660 \mathrm{~nm}$ and particle positions in the $x y$ plane were recorded by a topview video camera (Photron FASTCAM 1024 PCI) with a matching bandpass filter at a speed of 60 frames per second (fps). This laser diode can be moved in the vertical direction to perform a scan. The wavelength of the vertical laser sheet was $635 \mathrm{~nm}$ and the particle positions were recoded by a SONY XC-ST 50 video camera at a speed of 30 fps.

Using the top-view camera, we recorded a circular particle cloud where individual particles (with a total number of approximately 4000) can be identified and tracked in consecutive images. The cloud can be divided into two regions. In the central region, MF and PS particles are well mixed upon fast cooling, with most of the particles being in an amorphous state. In the outer region, only MF particles are present, forming hexagonal lattice structure surrounding the mixture. In the present paper, we focus on this mixture in the center. As we can see in Fig. 2(a), at certain locations, we observe localized ordered lattice domains of limited size. These domains have a square lattice, different from the typical hexagonal lattices in one-component complex plasma. In order to identify such structure, we define a local order parameter
$\Psi_{8}(\boldsymbol{r})$ for a particle with coordinate $\boldsymbol{r}$ as

$$
\Psi_{8}(\boldsymbol{r})=\frac{1}{8} \sum_{m} e^{i 8 \theta_{m}}
$$

where we only consider eight nearest neighbors and $\theta_{m}$ is the angle between $\boldsymbol{r}_{m}-\boldsymbol{r}$ and the $x$ axis, see Fig. 1 . The result is shown in Fig. 2(a). One can see a few squarelattice domains of various sizes embedded in the amorphous binary mixture. The largest domain is located at the left lower corner, comprised of approximately 80 particles. The mean interparticle distance of $a \approx 0.6 \mathrm{~mm}$ is deduced from the pair correlation function, shown as black squares in Fig. 2(d).

Using the side view camera, two layers of particles are recorded. Their height difference is about $0.14 \mathrm{~mm}$, as shown in Fig. 3, which is much smaller than the horizontal interparticle distance. The system can therefore be regarded as q2D. Furthermore, the particle motion in the vertical direction is barely visible with the used discharge power settings, and therefore we neglect this component in the analysis.

In order to identify the composition of this square lattice, we performed a vertical scan in a separate experimental run. The horizontal laser sheet moved down. During this procedure, the laser sheet first crosses the upper layer of MF particles (the purple dashed-dotted line in Fig. 3(c) represents a Gaussian fit of the recored particle image brightness), and then the lower layer of PS particles (the green dashed line). The MF and PS particles can thus be distinguished, and their structure is shown in Fig. 3(d). Note that the square lattice is slightly distorted, presumably due to the tension in the cloud.

To identify the collective dynamics of such square lattice, we computed the wave spectra using Fourier transformation. Here, we do not apply external excitation, and thus the natural phonon spectra are obtained. The wave spectra $\boldsymbol{V}_{\boldsymbol{k}, \omega}$ are calculated based on the particle velocity $\boldsymbol{v}(\boldsymbol{r}, t)$ :

$$
\boldsymbol{V}_{\boldsymbol{k}, \omega}=2 / P A \int_{0}^{P} \int_{0}^{A} \boldsymbol{v}(\boldsymbol{r}, t) \exp [-i(\boldsymbol{k} \cdot \boldsymbol{r}-\omega t)] d \boldsymbol{r} d t
$$

where $A$ and $P$ are the linear size of the area and the period over which the particle motion is summed. The spectra are calculated in the directions of $\theta=0^{\circ}$ and $45^{\circ}$ for both longitudinal $(\mathrm{L})$ and transverse $(\mathrm{T})$ mode ${ }^{38}$. The results are shown in Fig. 4.

To corroborate the experimental results, we performed a Langevin dynamics simulation. The equation of motion including damping reads

$$
m_{i} \ddot{\boldsymbol{r}}_{i}+m_{i} \nu \dot{\boldsymbol{r}}_{i}=\sum_{j} \boldsymbol{F}_{j i}+\boldsymbol{F}_{c, i}+\boldsymbol{L}_{i}
$$

where $\boldsymbol{r}_{i}$ is the particle position, $m_{i}$ the mass, $\nu_{i}$ the damping rate, $\boldsymbol{L}_{i}$ the Langevin heat bath. We use the same values of masses and damping rates as in the 

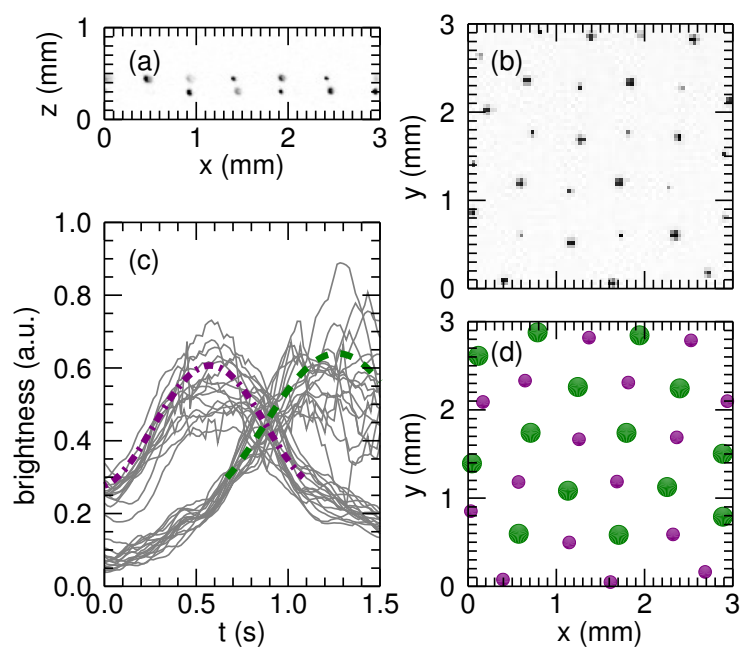

FIG. 3. Side view (a) and top view (b) of a q2D binary complex plasma recorded by the video cameras (inverted gray scale). The brightness of each particle, recorded by the topview camera during the scan from top to bottom, is shown in (c) by gray lines. Different particle types in (d) are indicated as in Fig. 1.

experiment. The Langevin force $\boldsymbol{L}_{i}(t)$ is defined by $<\boldsymbol{L}_{i}(t)>=0$ and $\left\langle\boldsymbol{L}_{i}(t) \boldsymbol{L}_{i}(t+\tau)\right\rangle=2 \nu_{i} m_{i} T \delta(\tau) \mathbf{I}$, where $T$ is the temperature of the heat bath, $\delta(\tau)$ is the delta function and $\mathbf{I}$ is the unit matrix.

We simulated a binary mixture of microparticles confined in the (pre)sheath of a plasma, where the gravitational force is balanced by the electrostatic force of the sheath field ${ }^{26,29,30}$. Since the two types of particles have different masses, their equilibrium heights deviate by $\Delta=0.14 \mathrm{~mm}$, same as in the experiment. We model this strong vertical confinement with a parabolic confinement, $\boldsymbol{F}_{c, i}=-C\left(z_{i}-z_{i, 0}\right) \boldsymbol{e}_{z}$, where $z_{i, 0}$ is the equilibrium position for particle $i$. In this paper, we focus on the inplane dynamics and thus suppress the vertical motion by setting $C=1 \mu \mathrm{N} / \mathrm{mm}$. In the horizontal direction, we apply periodic boundary conditions.

In the simulation, individual microparticles are modeled as negative point-like charges. These negatively charged particles distort the ion flow in the sheath, resulting in the ion wake below individual particles. This wake effect has been studied using various theoretical models such as Yukawa point-wake model ${ }^{21,39}$, dipole-wake mode $^{40}$, self-consistent model ${ }^{41,42}$, as well as particle-incell simulations ${ }^{43,44}$. Here, we apply the Yukawa pointwake model to include the ion wake in the particle interaction. This treatment is simple but can capture the essence of the underlying physics. In practice, a positive point-like charge is placed at a fixed vertical distance $\delta$ below each particle ${ }^{45,46}$. The force exerted on particle $i$ by particle $j$ is composed of two components: the repulsive force $\boldsymbol{F}_{j i}^{p}$ by particle $j$ and the attractive force $\boldsymbol{F}_{j i}^{w}$ by the point-like wake charge below particle $j$. Both components have a form of Yukawa interaction. The net effective force can be written as

$$
\boldsymbol{F}_{j i}=\boldsymbol{F}_{j i}^{p}+\boldsymbol{F}_{j i}^{w}=Q_{i} Q_{j} f\left(r_{j i}\right) \frac{\boldsymbol{r}_{j i}}{r_{j i}}+Q_{i} q_{j} f\left(r_{j i}^{w}\right) \frac{\boldsymbol{r}_{j i}^{w}}{r_{j i}^{w}},
$$

where $f(r)=\exp (-r / \lambda)(1+r / \lambda) / r^{2}$ with $\lambda$ being the screening length, $\boldsymbol{r}_{j i}=\boldsymbol{r}_{i}-\boldsymbol{r}_{j}$ and $\boldsymbol{r}_{j i}^{w}=\boldsymbol{r}_{i}-\left(\boldsymbol{r}_{j}-\right.$ $\left.\delta \boldsymbol{e}_{z}\right)$. Thus the interaction is non-reciprocal ${ }^{28,47,48}$. The molecular dynamics simulations were performed using LAMMPS in NVT ensemble ${ }^{49}$. The parameters were chosen according to the experimental values ${ }^{17,29}$. The charges for PS and MF particles are set to $Q_{\mathrm{PS}}=19000 e$ and $Q_{\mathrm{MF}}=15000 \mathrm{e}$, respectively, and the screening length is $\lambda=600 \mu \mathrm{m}$. The distance between the pointlike wake charge and the particle is set to $\delta=180 \mu \mathrm{m}$, i.e., $\delta / \lambda=0.3^{21,40,45}$. The total number of particles in the simulation is 6400 .

The formation of square-lattice domains depends not only on the particle size and plasma parameters, but also on the preparation of the particle cloud. In the experiments, the particles are compressed into a q2D layer by the increase of discharge power. In a domain of square lattice, MF and PS particles are arranged alternatively, as shown in Fig. 3(d). The preparation of binary complex plasma is a complicated procedure, which is beyond the scope of this paper and will be published elsewhere. In order to simulate the structure observed in the experiment, we arrange two types of particles alternatively, with an uncertainty of $30 \%$ of the interparticle distance as the initial condition. The system evolves for $10 \mathrm{sec}-$ onds to reach a steady state. The resulting structure is shown in Fig. 2(b). Though more particles show local square structure than in the experiment, the distribution of $\left|\Psi_{8}\right|$ in the simulation quantitatively agrees with that in Fig. 2(c).

In Fig. 4, we plot the dispersion relations of the inplane modes in the domain of square lattice, marked in Fig. 2(a). The two principal axes for both longitudinal and transverse mode spectra are chosen as $\theta=0^{\circ}$ and $45^{\circ}$ according to the intrinsic symmetry of the square lattice. The wave energy is concentrated along distinct curves in the $k-\omega$ space. The results are compared with the numerical simulation, from the domain marked in Fig. 2(b $)^{50}$. Since the domain size is limited, the spectra have a relatively high level of noise. Nevertheless, the simulation with selected parameters shows a fair agreement with the experiment when the wake charge is set to $20 \%$ of the particle charge, i.e., for $q / Q=0.2$.

In order to study the influence of the wake charge on the dispersion relations, we run the simulations for three wake charge ratios $(q / Q=0.2,0.4,0.6$, for which a square lattice can be formed) and plot the resulting phonon spectra in Fig. 5. To gain better resolution, here we initially arrange the particles in a staggered square lattice in the whole simulation area. The square-lattice structure is sustained after the relaxation.

Fig. 5 shows that the frequency of the longitudinal component decreases with the increase of the wake charge while that of the transverse component increases. The 

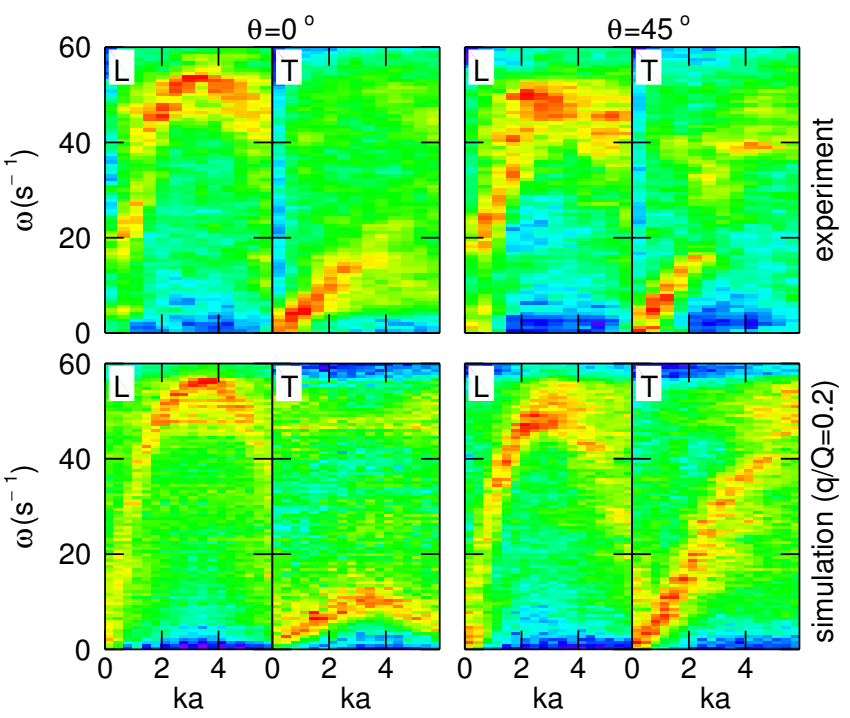

FIG. 4. Phonon spectra of the q2D binary complex plasma in the experiment (upper panels) and simulation (lower panels). The left and right columns show the spectra of longitudinal (L) and transverse $(\mathrm{T})$ modes at $\theta=0^{\circ}$ and $\theta=45^{\circ}$, respectively.

trend is particularly evident for the transverse component with $\theta=0^{\circ}$. These results can be directly compared with the experimental measurements, so that the value of $q / Q$ can be inferred ${ }^{51}$. However, sufficiently large domains should be found for this in experiments, so that clear spectra with detailed features can be obtained.

To summarize, this paper reports on the first study of domains of square lattice embedded in a disordered q2D binary complex plasma. We focus on the phonon spectra of the longitudinal and transverse in-plane modes. The experimental results are compared with the Langevin dynamics simulations, showing a good agreement. Furthermore, we demonstrate the strong effect of the wake charge on the shape of the spectra. We expect that the presented results will stimulate further studies of phonon spectra in binary complex plasmas.

\section{ACKNOWLEDGMENTS}

The authors acknowledge support from the National Natural Science Foundation of China (NSFC), Grant No. 11405030. We are thankful for support of this work by the Deutsche Forschungsgemeinschaft (DFG) through the grants IV 20/3-1. We thank I. Laut for valuable discussion.

\section{Appendix A: Wave spectra of a hexagonal lattice}

For comparison with the square-lattice domains, we select an area in the outer region of the particle cloud in the
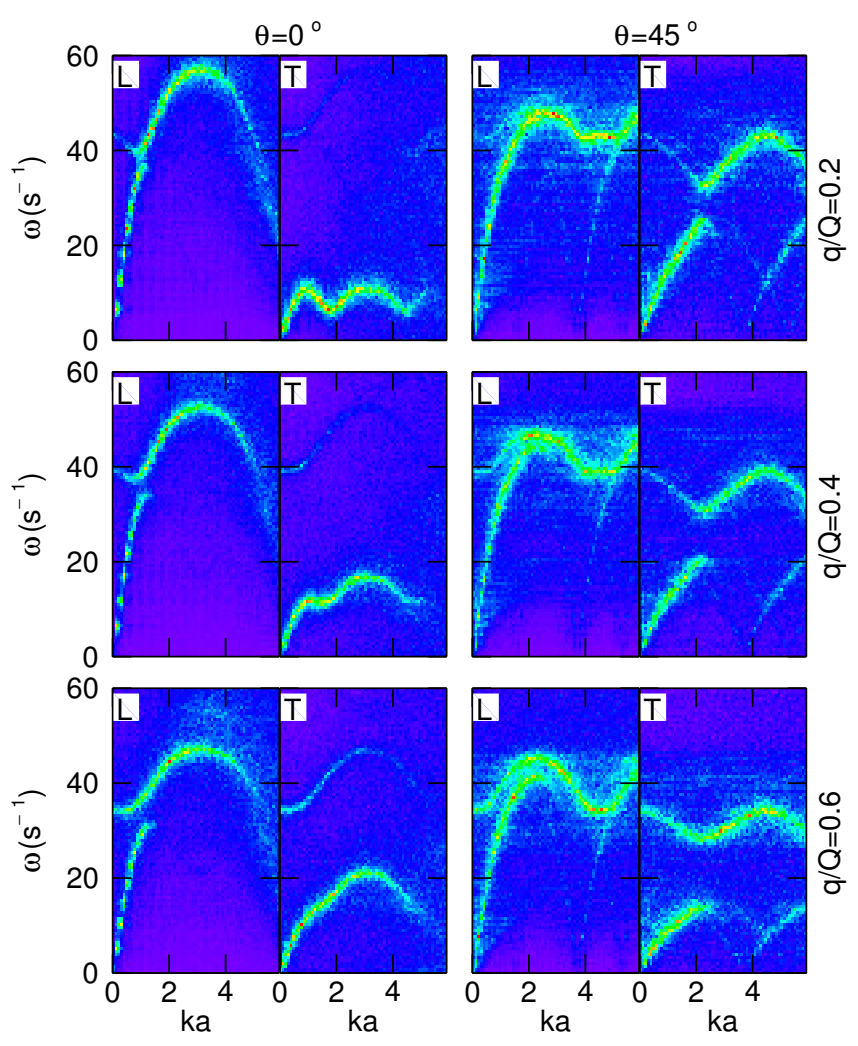

FIG. 5. Phonon spectra of the q2D binary complex plasma in the simulations. To enhance the quality of the spectra, here we use a larger number of particles compared to the experiment (4450 instead of 88). From top to bottom, the panels correspond to the ratio $q / Q$ of $0.2,0.4$, and 0.6 . The left and right columns show the spectra of the longitudinal (L) and transverse $(\mathrm{T})$ modes at $\theta=0^{\circ}$ and $\theta=45^{\circ}$, respectively. Here, we use only those values of $q / Q$ for which a square lattice can be formed.

same experimental run. This area is close to the periphery of the particle cloud and mainly contains MF particles. Here, the interparticle distance is slightly larger than that in the center part of the particle cloud. In this area, the particles form a triangular lattice with hexagonal symmetry, as often seen in one-component 2D plasma crystals $^{17,18,20}$. It is conventional to select $\theta=0^{\circ}$ and $\theta=30^{\circ}$ as principal axes to perform spectral analysis. For the purpose of direct comparison, we also include the spectra of the longitudinal and transverse modes for $\theta=45^{\circ}$. The results are shown in Fig. 6. Both modes show a different dispersion relation from those of a square lattice. Note that this selected domain is slightly larger than the one marked in Fig. 2(a), resulting in spectra with better resolution.

\footnotetext{
${ }^{1}$ J. H. Chu and L. I, Phys. Rev. Lett. 72, 4009 (1994).

${ }^{2}$ H. Thomas, G. E. Morfill, V. Demmel, J. Goree, B. Feuerbacher, and D. Möhlmann, Phys. Rev. Lett. 73, 652 (1994).

${ }^{3}$ Y. Hayashi and K. Tachibana, Japanese Journal of Applied Physics 33, L804 (1994).
} 

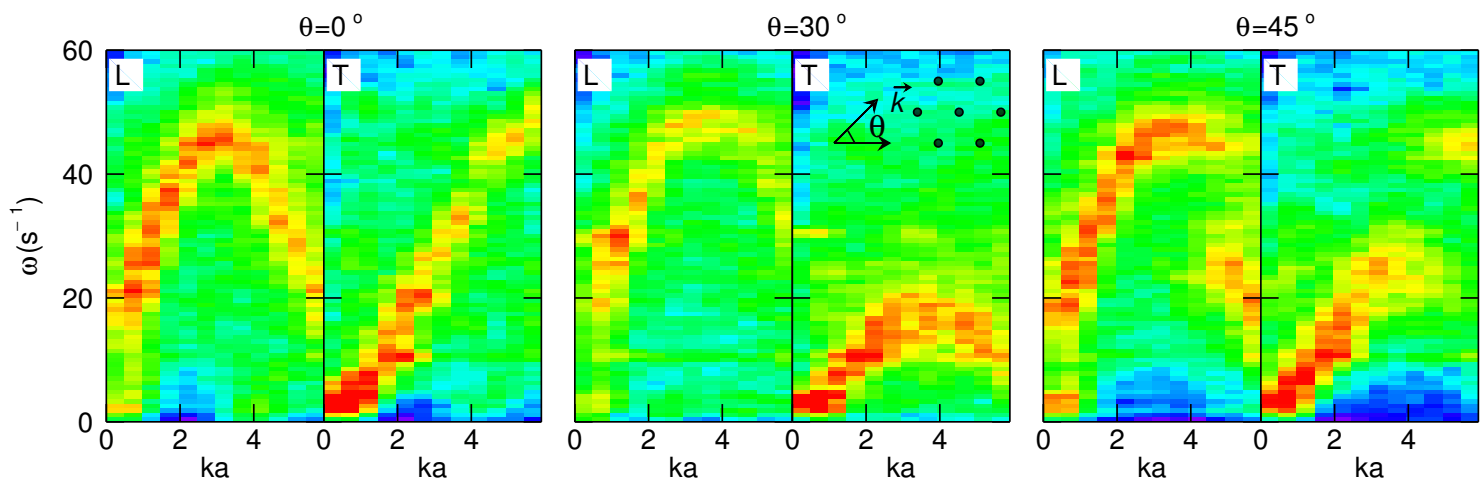

FIG. 6. Phonon spectra of hexagonal lattice in the same experimental run as shown in Fig. 4. This domain of hexagonal lattice is located close to the periphery of the particle cloud.

${ }^{4}$ A. Melzer, T. Trottenberg, and A. Piel, Physics Letters A 191, 301 (1994)

${ }^{5}$ G. E. Thomas, H. M.; Morfill, Nature 379, 806 (1996).

${ }^{6}$ V. Fortov, A. Ivlev, S. Khrapak, A. Khrapak, and G. Morfill, Physics Reports 421, 1 (2005).

${ }^{7}$ G. E. Morfill and A. V. Ivlev, Rev. Mod. Phys. 81, 1353 (2009).

${ }^{8}$ M. Chaudhuri, A. V. Ivlev, S. A. Khrapak, H. M. Thomas, and G. E. Morfill, Soft Matter 7, 1287 (2011).

${ }^{9}$ V. Nosenko, S. Zhdanov, and G. Morfill, Phys. Rev. Lett. 99, 025002 (2007).

${ }^{10}$ Y. Feng, J. Goree, and B. Liu, Phys. Rev. Lett. 100, 205007 (2008).

${ }^{11}$ C.-S. Wong, J. Goree, Z. Haralson, and B. Liu, Nature Physics 14, 21 (2018).

${ }^{12}$ P. Hartmann, G. J. Kalman, Z. Donkó, and K. Kutasi, Phys. Rev. E 72, 026409 (2005).

${ }^{13}$ C. A. Knapek, D. Samsonov, S. Zhdanov, U. Konopka, and G. E. Morfill, Phys. Rev. Lett. 98, 015004 (2007).

${ }^{14}$ P. Hartmann, A. Douglass, J. C. Reyes, L. S. Matthews, T. W. Hyde, A. Kovács, and Z. Donkó, Phys. Rev. Lett. 105, 115004 (2010).

${ }^{15}$ D. Samsonov, J. Goree, Z. W. Ma, A. Bhattacharjee, H. M. Thomas, and G. E. Morfill, Phys. Rev. Lett. 83, 3649 (1999).

${ }^{16}$ A. Melzer, S. Nunomura, D. Samsonov, Z. W. Ma, and J. Goree, Phys. Rev. E 62, 4162 (2000).

${ }^{17}$ C.-R. Du, V. Nosenko, S. Zhdanov, H. M. Thomas, and G. E. Morfill, EPL (Europhysics Letters) 99, 55001 (2012).

${ }^{18}$ S. Nunomura, J. Goree, S. Hu, X. Wang, A. Bhattacharjee, and K. Avinash, Phys. Rev. Lett. 89, 035001 (2002).

${ }^{19}$ S. Nunomura, J. Goree, S. Hu, X. Wang, and A. Bhattacharjee, Phys. Rev. E 65, 066402 (2002).

${ }^{20}$ J. K. Meyer, I. Laut, S. K. Zhdanov, V. Nosenko, and H. M. Thomas, Phys. Rev. Lett. 119, 255001 (2017).

${ }^{21}$ S. K. Zhdanov, A. V. Ivlev, and G. E. Morfill, Physics of Plasmas 16, 083706 (2009)

${ }^{22}$ L. Couëdel, V. Nosenko, A. V. Ivlev, S. K. Zhdanov, H. M. Thomas, and G. E. Morfill, Phys. Rev. Lett. 104, 195001 (2010).

${ }^{23}$ B. Liu, J. Goree, and Y. Feng, Phys. Rev. Lett. 105, 085004 (2010).

${ }^{24}$ B. Smith, T. Hyde, L. Matthews, J. Reay, M. Cook, and J. Schmoke, Advances in Space Research 41, 1510 (2008).

${ }^{25}$ C. Killer, T. Bockwoldt, S. Schütt, M. Himpel, A. Melzer, and A. Piel, Phys. Rev. Lett. 116, 115002 (2016).

${ }^{26}$ F. Wieben, J. Schablinski, and D. Block, Physics of Plasmas 24, 033707 (2017).

${ }^{27}$ A. Ivlev, H. Löwen, G. Morfill, and C. P. Royall, Complex Plasmas and Colloidal Dispersions: Particle-Resolved Studies of Classical Liquids and Solids (World Scientific, Singapore, 2012).
${ }^{28}$ A. V. Ivlev, J. Bartnick, M. Heinen, C.-R. Du, V. Nosenko, and H. Löwen, Phys. Rev. X 5, 011035 (2015).

${ }^{29}$ C.-R. Du, V. Nosenko, H. M. Thomas, G. E. Morfill, and A. V. Ivlev, "Slow dynamics in a quasi-two-dimensional binary complex plasma," (2016), arXiv:1609.01456.

${ }^{30}$ P. Hartmann, Z. Donkó, G. J. Kalman, S. Kyrkos, K. I. Golden, and M. Rosenberg, Phys. Rev. Lett. 103, 245002 (2009).

${ }^{31}$ G. J. Kalman, Z. Donkó, P. Hartmann, and K. I. Golden, Phys. Rev. Lett. 107, 175003 (2011).

${ }^{32}$ G. J. Kalman, P. Hartmann, Z. Donkó, K. I. Golden, and S. Kyrkos, Phys. Rev. E 87, 043103 (2013).

${ }^{33}$ E. C. Whipple, T. G. Northrop, and D. A. Mendis, Journal of Geophysical Research: Space Physics 90, 7405 (1985).

${ }^{34}$ P. S. Epstein, Physical Review 23, 710 (1924).

${ }^{35}$ B. Liu, J. Goree, V. Nosenko, and L. Boufendi, Physics of Plasmas 10, 9 (2003).

${ }^{36}$ V. Nosenko, A. V. Ivlev, and G. E. Morfill, Phys. Rev. Lett. 108, 135005 (2012).

${ }^{37}$ V. Nosenko, A. V. Ivlev, and G. E. Morfill, Phys. Rev. E 87, 043115 (2013).

${ }^{38}$ Each spectrum was calculated by analyzing the particle motions in the rectangle in Fig. 2(a) over a period of $8.5 \mathrm{~s}$ (510 frames) and then averaged over 16 periods to reduce the noise level.

${ }^{39}$ T. B. Röcker, A. V. Ivlev, S. K. Zhdanov, and G. E. Morfill, Phys. Rev. E 89, 013104 (2014).

${ }^{40}$ T. B. Röcker, A. V. Ivlev, R. Kompaneets, and G. E. Morfill, Physics of Plasmas 19, 033708 (2012).

${ }^{41}$ R. Kompaneets, U. Konopka, A. V. Ivlev, V. Tsytovich, and G. Morfill, Physics of Plasmas 14, 052108 (2007).

${ }^{42}$ R. Kompaneets, G. E. Morfill, and A. V. Ivlev, Phys. Rev. Lett. 116, 125001 (2016).

${ }^{43}$ W. J. Miloch, S. V. Vladimirov, H. L. Pécseli, and J. Trulsen, Phys. Rev. E 77, 065401 (2008).

${ }^{44}$ I. H. Hutchinson, Phys. Rev. E 85, 066409 (2012).

${ }^{45}$ I. Laut, S. K. Zhdanov, C. Räth, H. M. Thomas, and G. E. Morfill, Phys. Rev. E 93, 013204 (2016).

${ }^{46}$ I. Laut, C. Räth, S. K. Zhdanov, V. Nosenko, G. E. Morfill, and H. M. Thomas, Phys. Rev. Lett. 118, 075002 (2017).

${ }^{47}$ V. A. Schweigert, I. V. Schweigert, A. Melzer, A. Homann, and A. Piel, Phys. Rev. E 54, 4155 (1996).

${ }^{48}$ A. Melzer, V. A. Schweigert, and A. Piel, Phys. Rev. Lett. 83, 3194 (1999).

${ }^{49}$ S. Plimpton, Journal of Computational Physics 117, 1 (1995).

${ }^{50}$ The simulation area is larger than the one shown in Fig. 2(b). This domain is located far from the periodic boundary. Therefore the influence of the boundary condition can be neglected.

${ }^{51}$ The influence of the particle-wake distance $\delta$ on the wave spectra is negligible. 